\title{
La enseñanza de las matemáticas con el enfoque de la Teoría de Comunidades de Práctica
}

\section{Teaching mathematics from the perspective of the theory of Communities of Practice}

\author{
Isaias Miranda ${ }^{1}$ \\ Ana Luisa Gómez-Blancarte ${ }^{2}$
}

\begin{abstract}
Resumen: En este ensayo se argumenta que la Teoría de Comunidades de Práctica (TCOP) y, en particular, que los conceptos de comunidad de práctica, negociación de significado, correduría y objetos limitáneos, son útiles para hacer análisis de la enseñanza de las matemáticas. La afirmación parte de dos supuestos: 1) los profesores de matemáticas, durante el ejercicio de su profesión, conforman una comunidad de práctica; 2) esta comunidad está conformada por cuatro prácticas: de enseñanza, de gestión, de capacitación y de academia. La TCoP permite examinar la práctica de enseñanza de las matemáticas en relación con las prácticas de gestión, de capacitación y de academia. Se argumenta que esta relación es posible al concebir al profesor como un corredor que incorpora, en su práctica de enseñanza, la información generada por los objetos limitáneos producidos en las prácticas de gestión, capacitación y academia. Sin embargo, esta relación solo es posible si el profesor negocia los significados de esos objetos limitáneos. El ensayo termina con una reflexión sobre cómo este análisis podría plantear nuevas preguntas sobre el tipo de comunicación que debe propiciar el profesor dentro del aula.
\end{abstract}

Fecha de recepción: 12 de diciembre de 2017. Fecha de aceptación: 25 de octubre de 2018.

1 Instituto Politécnico Nacional, CICATA-Legaria. imirandav@ipn.mx orcid.org/0000-0003-2076-7383.

2 Instituto Politécnico Nacional, CICATA-Legaria. algomezb@ipn.mx orcid.org/0000-0002-8837-8643. 
Palabras clave: Enseñanza de las matemáticas, profesor de matemáticas, teoría social del aprendizaje, comunidades de práctica.

\begin{abstract}
In this essay we propose an analysis of the teaching of mathematics based on the concepts of community of practice, negotiation of meaning, brokering, and boundary objects, which emerged in the Theory of Communities of Practice. The analysis is based on two assumptions: 1) mathematics teachers form a community of practice during their professional practice; 2) this community is made up of four practices, identified as: teaching, management, training, and academia. Thus, the proposal is to examine the practice of mathematics teaching in relation to management, training, and academia. It is argued that this relationship is possible by conceiving the teacher as a broker who incorporates the information generated by the boundary objects produced in management, training, and academic practices into his or her teaching practice. It is proposed that this relationship is only possible if the teacher negotiates the meanings of these boundary objects. It ends with a reflection on how this analysis might raise new questions about the type of communication the teacher should encourage in the classroom.
\end{abstract}

Keywords: Mathematics teaching, mathematics teacher, social theory of learning, communities of practice.

\title{
INTRODUCCIÓN
}

Uno de los principales objetivos de la investigación en educación matemática es proporcionar fundamentos teóricos con los que pueda estudiarse la enseñan$z^{3}{ }^{3}$ de las matemáticas y, con ello, sugerir estrategias para mejorar el aprendizaje. Los desarrollos de la Teoría de Situaciones Didácticas (TSD) (Brousseau, 1997) y de la Teoría Antropológica de lo Didáctico (TAD) (Chevallard, 1999) son, quizá, los ejemplos más representativos de este objetivo. Ambas teorías tienen la

3 En este ensayo aceptamos la definición de enseñanza de Gage (2009) según la cual esta es la influencia que ejerce una persona para mejorar el aprendizaje de otras. La enseñanza de las matemáticas es, entonces, un ejemplo de enseñanza. Aquí se hace un uso indistinto entre enseñanza y enseñanza de las matemáticas. 
intención de estudiar las condiciones con las que el profesor puede lograr que el conocimiento a enseñar sea el resultado de la interacción entre el profesor, sus estudiantes y el saber matemático -en el caso de la TSD-, y entre el profesor, sus estudiantes, las instituciones educativas y las empresas del sector industrial -en el caso de la TAD-. Sin embargo, el estudio de la enseñanza no solo se enfoca en el análisis de estas condiciones. Por ejemplo, con su Teoría de la Enseñanza-en-Contexto (TECo), Schoenfeld (1998) pretende identificar el tipo de variables que intervienen en las decisiones del profesor en el momento de impartir sus clases.

Una característica común de la TSD, la TAD y la TECo es que los principios teóricos en los que basan sus análisis pertenecen a paradigmas (en el sentido de Kuhn (1971)) educativos. Por un lado, la TECo se basa en el paradigma cognitivo de Leinhardt y Greeno (1986), quienes suponen que la enseñanza es una habilidad cognitiva que permite al profesor tomar decisiones en el salón de clase. Por otro, la TSD y la TAD desarrollan sus propuestas de enseñanza a partir de los principios constructivistas de los paradigmas psicogenético de Piaget (1948) y sociocultural de Vygotsky (1978) sobre el aprendizaje (véase la categorización de paradigmas en psicología de la educación hecha por Hernández-Rojas (1998).

Aunque los paradigmas cognitivo, psicogenético y sociocultural sobre el aprendizaje humano han aportado elementos metodológicos y teóricos útiles al estudio de la enseñanza de las matemáticas, algunos investigadores aseguran que estos elementos no son suficientes. Esta insuficiencia se debe a varias razones. Por un lado, a la diversidad de variables que deben tomarse en cuenta en el estudio de la enseñanza de las matemáticas (Adler, Ball, Krainer, Lin \& Novotna, 2005) y la variedad de preguntas de investigación que las teorías desean responder (incluso de aquellas teorías que parten del mismo paradigma; véase Rogalski, (2003, p. 345)). Por otro lado, al modo como en esos paradigmas se elaboran los constructos teóricos con los que se estudia la enseñanza (véanse las críticas de Roth y Radford (2010) al concepto de Zona de Desarrollo Próximo; y la de Skott, Moeskaer Larsen y Hellsten Østergaard (2011) al paradigma cognitivo).

Una posible alternativa para superar las insuficiencias mencionadas es utilizar un paradigma social en el análisis de la enseñanza de las matemáticas. En este artículo se propone la utilización de este paradigma y, en particular, de la Teoría de Comunidades de Práctica (TCoP) (Wenger, 2001), para explorar cómo puede caracterizarse la enseñanza. La propuesta se fundamenta tanto en la sugerencia de Morgan (2014) sobre la necesidad de comprender las prácticas individuales a partir del modo como los individuos se relacionan con prácticas inmersas en estructuras 
sociales más amplias como en la recomendación de Skott (2013) acerca de estudiar las prácticas que el profesor realiza dentro del salón de clase, tomando en cuenta el constante cambio de sus formas de participar en diferentes prácticas sociales 4 .

La TCoP ha sido utilizada con frecuencia en estudios de educación en general (Buysse, Sparkman, \& Wesley, 2003) y de educación matemática en particular (Groth, 2015). Al igual que la teoría de Piaget y la de Vygotsky, la TCoP fue creada para explicar el aprendizaje ocurrido fuera de los ambientes escolares. De hecho, en la introducción de su libro Comunidades de práctica: aprendizaje, significado e identidad, Wenger (2001) asegura que en su teoría la concepción de aprendizaje no está relacionada con entornos escolares, sino con situaciones surgidas en la vida diaria. Sin embargo, a diferencia de las dos primeras teorías en las que el aprendizaje es interpretado como la transformación de las estructuras cognitivas del individuo por medio de la forma en que asimila y acomoda la información externa (Pozo, 1989), en la TCoP se desarrolla una comprensión de la naturaleza social del aprendizaje humano (Wenger, 2010). Aun cuando la teoría vygotskyana le da importancia al aspecto social del aprendizaje, existe una diferencia entre esta y la TCoP en cuanto a los conceptos que tratan de dar cuenta de este aspecto: mientras que el concepto medular en el paradigma sociocultural de Vygotsky es el de actividad (Kozulin, 2000), en el paradigma social de Wenger el concepto fundamental es el de comunidad de práctica.

Tomando en cuenta los resultados de los paradigmas psicogenético y sociocultural, en la primera sección se describe cómo algunos autores han concebido la enseñanza en estos paradigmas. La decisión de incorporar esta descripción se debe a que con ella se ofrece un punto de referencia alrededor del cual puede ponerse en perspectiva la interpretación de la TCoP hecha en este escrito. En la segunda sección, que parte del supuesto de que la enseñanza es una práctica, en el sentido que se le da a este término en la TCoP, se argumenta cómo por medio de la Teoría de Comunidades de Práctica y de los conceptos de negociación de significado, correduría y objetos limitáneos se puede estudiar esta práctica. Por último, en la tercera sección se mencionan algunas reflexiones sobre las implicaciones didácticas que resultan de analizar la enseñanza con los conceptos de la TCoP.

4 Skott (2013) utiliza el término "práctica" para referirse a las acciones que el profesor realiza dentro y fuera de su salón de clase. Así, por ejemplo, él identifica que algunas prácticas del salón de clase se relacionan con las matemáticas (por ejemplo, la enseñanza de un contenido específico) y otras no (por ejemplo, controlar la disciplina). En lo que se refiere a las prácticas fuera del salón de clase, pueden ser la vida personal y el trabajo con colegas. 


\section{LA ENSEÑANZA DE LAS MATEMÁTICAS EN LOS PARADIGMAS PSICOGENÉTICO Y SOCIOCULTURAL: UN BREVE ANÁLISIS}

Piaget y Vygotsky parten del supuesto de que el aprendizaje es un proceso organizado y estructurado (Pozo, 1989); precisamente por esta coincidencia es posible encontrar similitudes entre las líneas de investigación desarrolladas por ambos (Cole \& Wertsch, 2000; DeVries, 2000; Shayer, 2003). Sin embargo, existen diferencias sutiles en cuanto a las implicaciones que ese supuesto tiene en la forma de concebir la enseñanza de las matemáticas.

El paradigma psicogenético sugiere que los profesores son productores de secuencias didácticas con las que los estudiantes pueden construir su propio conocimiento. Esto no quiere decir que la enseñanza tenga un papel secundario en el aprendizaje (Lerner, 1996). Para Piaget (1948), la enseñanza es un espacio donde el profesor crea problemas útiles para los estudiantes. Por "útiles", Piaget se refiere a aquellos problemas que estimulan la iniciativa y la investigación en los estudiantes. En este sentido, el profesor piagetiano no es un espectador del aprendizaje; debe, por el contrario, inducir a los estudiantes a re-descubrir el significado de los conceptos (Piaget, 1948, pp. 19 y sigs.). Esto significa que, en la concepción de enseñanza piagetiana, el profesor es un agente activo que toma en cuenta el hecho de que cada estudiante estructura sus propios esquemas (Webb, 2001). De esta forma, el profesor debe considerar que el aprendizaje es una reestructuración activa del pensamiento individual y no un incremento conceptual del contenido de los temas que enseña; en este sentido, es deseable que el profesor individualice "las experiencias de aprendizaje de tal forma que cada estudiante trabaje a un nivel suficientemente alto para ser un reto y suficientemente realista para prevenir una frustración excesiva" (Webb, 2001, p. 97). En otras palabras, en la enseñanza constructivista de Piaget el profesor propone problemas que permiten que cada estudiante reconstruya los contenidos escolares (Lerner, 1996).

En el paradigma sociocultural de Vygotsky (1978) la enseñanza no solo es un espacio propicio donde el profesor puede generar oportunidades para que los estudiantes redescubran los conceptos, sino también uno en el que el profesor promueve el desarrollo conceptual de los estudiantes (Clarà, 2017). Vygotsky argumenta que al niño no se le debería (y no se le podría) pedir que comprenda el mundo por medio del descubrimiento de las principales leyes explicativas ya descubiertas por la humanidad. El desarrollo del niño es tan especial, en gran parte, debido a que los adultos les enseñan esas leyes ya 
elaboradas por la comunidad científica (Karpov \& Haywood, 1998). De hecho, la relevancia que tiene la enseñanza en el mejoramiento del aprendizaje se explica a través de la aplicación inmediata de los resultados de Vygotsky. Según estos, el rol del adulto es determinante en el manejo que hagan los niños de instrumentos físicos o simbólicos para ejecutar con eficiencia operaciones psicológicas específicas, tales como la memoria, la atención y el lenguaje. De acuerdo con Vygotsky, un profesor no solo guía a los estudiantes en la construcción de su conocimiento, sino que forma parte de esta construcción. El concepto de Zona de Desarrollo Próximo fue creado por Vygotsky precisamente para analizar el tránsito del nivel (actual) de desarrollo conceptual que tienen los estudiantes antes de la instrucción al nivel (potencial) de desarrollo que ellos pueden alcanzar después de la misma. Este concepto, además de ser uno de los más importantes en la teoría de Vygotsky, es uno de los más utilizados y modificados por los investigadores para explorar los fenómenos educativos relacionados con la enseñanza de las matemáticas (Norton \& D'Ambrosio, 2008; Roth \& Radford, 2010).

Aun cuando los paradigmas piagetiano y vygotskiano difieran en las preguntas básicas que cada uno de ellos se formula (Castorina, 1996), es posible encontrar similitudes en cuanto a la aplicación de sus resultados en la conceptualización de la enseñanza de las matemáticas. Así, lo que Hedegaard (1990) sugiere respecto de lo que deberá hacer una enseñanza basada en el paradigma sociocultural puede aplicarse a una enseñanza basada en el paradigma psicogenético: en ambos paradigmas es posible identificar que la enseñanza puede organizarse con tres acciones principales: "(1) delineación del problema; (2) solución del problema y construcción del problema (el cual implica la adquisición de capacidades); (3) evaluación y control" (Hedegaard, 1990, p. 357). La sugerencia de Hedegaard ejemplifica la característica principal de los esfuerzos de los investigadores por vincular los aportes de estos paradigmas con la enseñanza de las matemáticas: ofrecer a los profesores una guía con la que ellos puedan organizar sus clases.

Una de las consecuencias de esta vinculación es el análisis que se hace de la enseñanza de las matemáticas en el contexto de las interacciones sucedidas en el aula. Este análisis ha contribuido significativamente a conocer la naturaleza del fenómeno educativo de la enseñanza de las matemáticas escolares y ha permitido proponer que las dinámicas ocurridas en el salón de clase son únicas e irrepetibles (ver los trabajos de Hammer (1995) 
y Walshaw (2008)) 5 . Sin embargo, en algunas investigaciones (Cobb, McClain, Lamberg \& Dean, 2003; Peressini, Borki, Romagnano, Knuth \& Willis, 2004) se sugiere estudiar la enseñanza no solo en la interacción del aula (es decir, entre el profesor y sus estudiantes), sino en relación con lo que ocurre fuera de ella. Por ejemplo, tomando como marco teórico la TCoP, en la investigación de Cobb et al. (2003) se describe la práctica de la enseñanza de las matemáticas dentro de los escenarios institucionales de las escuelas en los que dicha práctica sucede.

En la siguiente sección exponemos cómo a partir de un paradigma social podría ser estudiada la interacción que inevitablemente el profesor establece con sus colegas. La tesis principal que se sostiene es considerar a la enseñanza de las matemáticas como una práctica que forma parte de una comunidad. Argumentamos que esta comunidad se identifica por varias prácticas en las que el profesor de matemáticas se involucra en el momento de ejercer su trabajo profesional.

\section{LA ENSEÑANZA DE LAS MATEMÁTICAS COMO PRÁCTICA DE UNA COMUNIDAD}

El análisis de la enseñanza de las matemáticas desde el punto de vista de la práctica en una comunidad se basa, principalmente, en la aplicación de tres conceptos (relacionados entre sí) de la TCoP: práctica, negociación de significado y comunidad de práctica. En los siguientes párrafos describimos el significado de estos conceptos, tal como son interpretados en este artículo, y la relación entre ellos. Primero se describe la relación entre el concepto de práctica y el de negociación de significado; después, la relación entre práctica y comunidad de práctica.

5 Hammer (1995), al observar que las sugerencias hechas en investigaciones relacionadas con la función del profesor no siempre son útiles para atender las situaciones vividas en los salones de clase, asegura que: "cada salón -y cada estudiante y cada profesor- es en un sentido único, y el modo como la instrucción se desarrolla depende de sus circunstancias particulares" (p. 408). Por su parte, Walshaw (2008), al asegurar que la labor del profesor es compleja, declara: "[lo] que funciona para un profesor y un grupo de estudiantes no necesariamente funciona para otro profesor con un grupo diferente de estudiantes" (p. 120). 


\section{PRÁCTICA Y NEGOCIACIÓN DE SIGNIFICADO: SU RELACIÓN EN LA ENSEÑANZA}

De acuerdo con Wenger (2001), la práctica es "hacer algo en un contexto histórico y social que otorga una estructura y un significado a lo que hacemos" (p. 71). La propuesta es, pues, considerar la enseñanza de las matemáticas como una práctica. Esta consideración puede justificarse en el hecho de que las acciones del profesor dentro del aula tienen sentido solo dentro de instituciones educativas que fueron creadas en momentos históricos específicos de la humanidad (ver Manacorda (1987) para conocer más detalles sobre estos momentos) y que, a través del tiempo, formaron parte del desarrollo cultural de las sociedades que las crearon. De esta forma, las acciones del profesor de matemáticas son significativas en la medida en que estén dirigidas a propiciar un aprendizaje escolar.

Este "hacer contextual" al que hace referencia la definición de práctica es el que permite que, aun cuando los seres humanos hagamos lo mismo una y otra vez, siempre podamos dar un nuevo significado a eso que hacemos. Es en este sentido en que es posible asegurar que un profesor de matemáticas, aunque enseñe el mismo tema en dos aulas, no interactúe de la misma forma con los estudiantes de ambos grupos, pues las dudas que pueden surgir en uno de ellos no necesariamente serán las mismas que surjan en el otro. Incluso si esas dudas son las mismas, las formas de aclararlas en ambos grupos serán distintas debido a las circunstancias específicas de cada grupo (por ejemplo, por el número de estudiantes) $)^{6}$. En términos de la teoría de Wenger, las distintas interacciones son causadas por las diferentes formas en que el profesor participa con cada uno de sus grupos. En efecto, dado que en la TCoP el concepto de participación se refiere "al proceso de tomar parte con otras personas y también a las relaciones con otras personas que reflejan este proceso" (Wenger, 2001, p. 80), el profesor establecerá relaciones distintas con los estudiantes de cada grupo a partir de la forma que él considere pertinente para atender las situaciones que surjan; pero precisamente esta forma específica de participación determina que el profesor utilice diferentes estrategias didácticas frente a cada grupo. Estas estrategias son ejemplos de lo que en la TCoP se conoce como el proceso de cosificación. Este proceso consiste en "dar forma a nuestra experiencia produciendo objetos que

6 En la investigación de Sensevy, Schubauer-Leoni, Mercier, Ligozat y Perrot (2005) se observa cómo dos profesores, usando la misma situación didáctica, no interactúan de la misma forma con sus respectivos estudiantes. 
plasman esta experiencia en una 'cosa'" (Wenger, 2001, p. 84). Esos objetos pueden ser instrumentos, símbolos, definiciones y conceptos. Según Wenger, la cosificación puede hacer referencia tanto a un proceso como a su producto, pues incluye procesos como hacer, percibir e interpretar.

Ahora bien, tanto el concepto de participación como el de cosificación se relacionan uno con el otro. Por ejemplo, ante la misma duda de estudiantes de dos grupos diferentes sobre si el dominio de la función $1 /\left(x^{\wedge} 2-4\right)$ es o no el conjunto de números reales, el proceso de participación del profesor, en un grupo, puede consistir en mostrar a todos los estudiantes una tabla que contenga los valores que adquiere la función conforme $x$ adquiere diferentes valores; en el otro grupo, el profesor puede pedir a cada estudiante que, con la ayuda de un programa de geometría dinámica, grafique la función y determine si es posible que se pueda obtener un valor de ella en los números 2 y -2. Estas maneras distintas de relacionarse (procesos de participación) con los estudiantes (una que requiere de la exposición verbal; otra que requiere de la atención individualizada) le dan forma, a su vez, a las estrategias didácticas (procesos de cosificación) del profesor. En otras palabras, al decidir cómo responderá la pregunta (es decir, al decidir cómo participar con sus estudiantes), el profesor determina su estrategia (es decir, el profesor cosifica su participación). Podríamos decir que, por un lado, la cosificación (por ejemplo, estrategias de enseñanza) permite que la participación (por ejemplo, acciones con las que se implementan esas estrategias) sea visible y, por otro, la participación permite que la cosificación sea interpretable. La relación entre estos dos procesos no significa que los conceptos de cosificación y participación se opongan uno al otro, sino que se enriquecen mutuamente (Farnsworth, Kleanthous \& Wenger-Trayner, 2016); forman una dualidad que Wenger (2001) llama negociación de significado. Debe tomarse en cuenta que esta dualidad no clasifica el significado en dos procesos distintos.

No es que cuando tengas más de uno [cosificación o participación], tienes menos de otro. Al contrario, la negociación de significado siempre involucra ambos Iprocesos] en una relación recíproca. De esta forma, siempre se tienen que buscar los dos procesos cada vez que se intente comprender un momento de producción de significado (Farnsworth et al., 2016, p. 146).

Para puntualizar la relación entre práctica y negociación de significado, retomemos el caso del profesor que enseña el mismo contenido a dos grupos distintos. Es en su práctica de enseñar -caracterizada por sus explicaciones verbales, lo 
que escribe en el pizarrón, etcétera- donde pueden observarse sus formas de negociar el significado de los conceptos matemáticos. Pero, ¿̇ué es lo que el profesor negocia durante su práctica de enseñanza? Para responder esta pregunta, debe primero recordarse que la TCoP es una teoría que enfoca su atención en explicar el aprendizaje de los individuos que pertenecen a una comunidad de práctica. Como los individuos que analizamos en esta propuesta son los profesores de matemáticas, el proceso de negociación de significado al que hacemos referencia se relaciona con lo que el profesor hace en otras prácticas. De hecho, el acercamiento analítico propuesto en este ensayo consiste en establecer una relación entre la práctica de enseñanza y esas otras prácticas. De esta forma, proponemos que la respuesta a la pregunta anterior sea que el profesor, al enseñar, re-negocia los significados producidos en prácticas generadas fuera de su salón de clase (ver Figura 1). Antes de describir las prácticas que hemos asociado con la práctica de enseñanza, expliquemos ahora la relación entre el concepto de comunidad de práctica y el de práctica. Esta relación permitirá enmarcar la práctica de la enseñanza y esas otras prácticas en una comunidad de práctica.

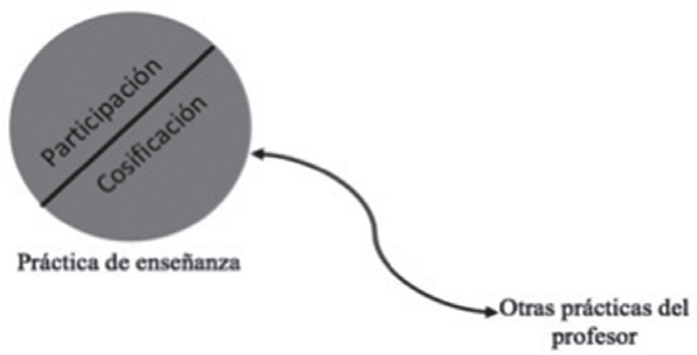

Figura 1. Esquema de la relación entre la práctica de enseñanza y otras prácticas del profesor.

\section{PRÁCTICA Y COMUNIDAD DE PRÁCTICA: SU RELACIÓN EN LA ENSEÑANZA}

El concepto de comunidad de práctica no se refiere a la aglomeración de individuos que hacen lo mismo para conseguir un fin común. Según Wenger (2001), 
una comunidad de práctica es la asociación de personas "que participan en acciones cuyo significado negocian mutuamente" (p. 100). Es esta negociación mutua la que permite que toda comunidad de práctica sea caracterizada por tres dimensiones: compromiso mutuo, empresa conjunta y repertorio compartido. Para ilustrar cada una de estas dimensiones, consideremos la comunidad de profesores de matemáticas. El compromiso mutuo que establecen los profesores que enseñan esta disciplina consiste no solo en implementar sus mejores estrategias didácticas para que sus estudiantes aprendan, sino también en compartir con ellos mismos el conocimiento que ellos tienen respecto de lo que saben y conocen de la enseñanza de las matemáticas. No se discute aquí cuál es la mejor estrategia para enseñar. Lo que se argumenta es que cualquiera que sea, cada profesor se compromete con sus colegas para que su enseñanza contribuya a una mejor comprensión de los conceptos. En la medida en que cada profesor, en su práctica de enseñanza, se esfuerce por lograr que todos sus estudiantes logren mejorar su aprendizaje, fortalecerá su compromiso con el resto de los profesores de matemáticas de su institución.

En cuanto a la dimensión de empresa conjunta, no se caracteriza por el hecho de que todos los profesores enseñen lo mismo, al mismo tiempo y de la misma manera. Esto, como ya se ha comentado, parece imposible (ver pie de página 3). La empresa conjunta de la comunidad de práctica de enseñanza de las matemáticas consiste en que cada profesor sabe que su práctica (la de enseñar) no solo es el producto de reflexiones individuales, sino también el resultado de haber negociado con sus compañeros cuáles pueden ser las formas más eficientes de hacer comprensibles los contenidos matemáticos; es decir, es el resultado de una negociación de significado sobre las formas de enseñar esos contenidos.

Ahora bien, para que esta empresa conjunta se logre es necesario que cada profesor comparta el mismo repertorio. La dimensión repertorio compartido incluye "rutinas, palabras, instrumentos, maneras de hacer, relatos, gestos, símbolos [...] acciones o conceptos que la comunidad ha producido o adoptado en el curso de su existencia y que han pasado a formar parte de su práctica" (Wenger, 2001, p. 110). El repertorio que comparten los profesores durante su práctica de enseñanza se puede observar, por ejemplo, en el hecho de que no todos los conceptos que ellos mencionan tienen que ser explicados: para todos ellos la palabra "estrategia didáctica" tiene un significado concreto dentro de su propia práctica de enseñanza. Ese significado común permite que esta palabra no tenga que ser explicada cada vez que sea mencionada por uno o por varios profesores. Lo mismo sucede con el uso de los instrumentos que definen su quehacer en esta 
comunidad: todos los profesores conocen, por ejemplo, la funcionalidad del pizarrón como un recurso que ayuda a organizar su discurso dentro del aula.

Volvamos ahora a la descripción de esas otras prácticas (ver Figura 1) en las que el profesor de matemáticas se involucra en el momento de ejercer su profesión como educador. De manera similar al análisis de Skott (2013), nosotros identificamos, además de la práctica de enseñanza, al menos tres tipos de prácticas en las que, voluntaria o involuntariamente, los profesores se involucran. A estas prácticas las hemos Ilamado práctica de academia, práctica de gestión y práctica de capacitación.

Así, en resumen, el análisis de la enseñanza que se propone en este ensayo consiste en enfocarse en la práctica de enseñar matemáticas tomando en cuenta que, entre ella y las prácticas de academia, de gestión y de capacitación, existe una relación. Ahora bien, todas estas prácticas en las que está involucrado el profesor deben concebirse como aquellas que suceden en una comunidad de práctica, cuyo principal compromiso, como ya se dijo antes, es el mejoramiento del aprendizaje del contenido de matemáticas expuesto en los planes y programas de estudio avalados por los centros educativos. Para poder explicitar cómo se establece esa relación, detallamos a continuación las características de las prácticas de academia, de gestión y de capacitación.

\section{PRÁCTICA DE ACADEMIA}

Si consideramos que el profesor de matemáticas pertenece a una academia que se reúne de manera periódica, es pertinente considerar que las decisiones del aula están influenciadas por las decisiones que se toman de forma colegiada respecto de los contenidos del currículo que deben ser enseñados, justamente en reuniones de academia. La práctica de academia puede observarse por lo que hace el profesor de matemáticas cuando se congrega con sus colegas para proponer soluciones a los problemas de aprendizaje que él observa en sus clases.

\section{PRÁCTICA DE GESTIÓN}

El profesor de matemáticas no solo colabora con sus colegas en las reuniones de academia, sino que también lo hace con las propias autoridades de la institución (ver el trabajo de Cobb et al. 2003). Las investigaciones de Hallinger y 
Heck (1998) confirman que las acciones realizadas por los directivos de las instituciones educativas afectan el desempeño de la enseñanza. Esto permite inferir que los intereses de las instituciones educativas podrían verse reflejados en la práctica de enseñanza. La práctica que el profesor lleva a cabo con sus autoridades, en la que discute y atiende las disposiciones oficiales que ellas emiten, es caracterizada como práctica de gestión.

\section{PRÁCTICA DE CAPACITACIÓN}

Con las constantes reformas educativas propuestas tanto por organismos internacionales como nacionales, las instituciones educativas solicitan a los profesores de matemáticas actualizar sus técnicas de enseñanza, o bien permitiéndoles asistir a cursos de capacitación docente (por ejemplo, diplomados, talleres), o bien motivándolos a realizar estudios de posgrado relacionados con la didáctica de las matemáticas. Esta práctica, denominada práctica de capacitación, consiste en la participación del profesor de matemáticas en acciones formativas que le otorguen conocimientos específicos para mejorar su enseñanza.

\section{LA RELACIÓN ENTRE LA PRÁCTICA DE ENSEÑANZA Y LAS PRÁCTICAS DE ACADEMIA, DE GESTIÓN Y DE CAPACITACIÓN}

Para comprender cómo se relaciona la práctica de enseñanza con lo que hemos denominado otras prácticas del profesor de matemáticas (de academia, de gestión y de capacitación), utilizamos los conceptos de objetos limitáneos y correduría. Con ellos, Wenger (2001), en su TCoP, pretende dar cuenta del modo como distintas prácticas se vinculan entre sí. El concepto de objeto limitáneo se usa para referirse a objetos que establecen conexiones cosificadoras porque "emplean formas inconexas de participación" (Wenger, 2001, p. 139). Pueden ser artefactos, documentos y aquellos objetos, tangibles o no, en torno a los cuales las comunidades de práctica organizan su interconexión. Un ejemplo de un objeto limitáneo es el escrito de una secuencia didáctica diseñada por un profesor durante su práctica de capacitación. La secuencia, producto del proceso de cosificación ocurrido en esta práctica, proporciona al profesor la oportunidad de iniciar (o de continuar) el proceso de participación en la práctica de enseñanza. El "empleo inconexo" del objeto limitáneo, 
mencionado en la definición anterior, se refiere a este hecho: al de ser cosificado en una práctica y utilizado para participar en otra.

El concepto de correduría se usa para un objeto que "ofrece una conexión participativa (...) porque lo que los corredores aportan para conectar prácticas es su experiencia de multi-afiliación y las posibilidades de negociación inherentes a la participación" (Wenger, 2001, p. 142). Continuando con el ejemplo de la secuencia didáctica, se puede argumentar que, si bien conecta la práctica de capacitación con la de enseñanza, dicha conexión estaría incompleta sin la participación del profesor, la cual consiste en darle significado a esa secuencia dentro de su enseñanza. Es en este sentido en que el profesor de matemáticas puede considerarse como un corredor.

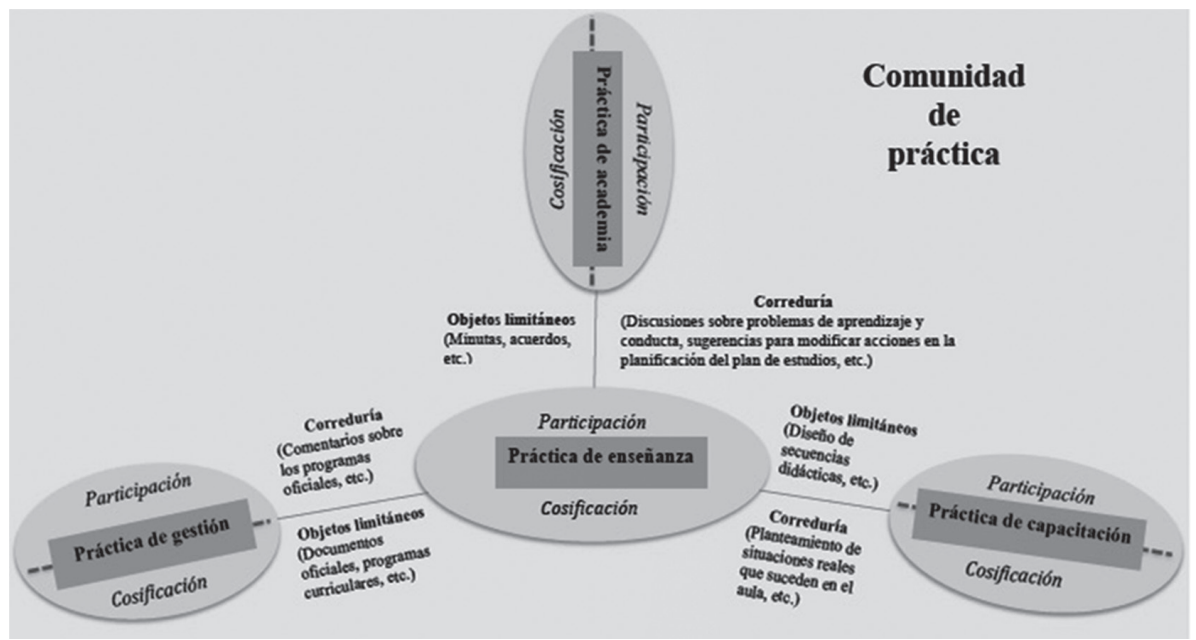

Figura 2. Esquema con el que se ilustra la relación entre la práctica de enseñanza y las prácticas de capacitación, de gestión y de academia.

En la figura 2 se ilustran los tipos de conexión y los procesos de participación y cosificación en la práctica de enseñanza y su relación con las otras prácticas mencionadas en uno de los extremos de la flecha de la figura 1. De esta forma, la figura 2 es una continuación de la figura 1.

En cada práctica (señaladas en óvalos) de la figura 2, el profesor negocia significados. Por sí misma, esta negociación no es suficiente para establecer la relación entre esa práctica y la de enseñanza. Es necesario que el profesor 
se involucre en la práctica de enseñanza -es decir, que actúe como corredorpara que esta se relacione con la otra práctica. Más claramente, las conexiones provocadas por los objetos limitáneos y la correduría se producen por la forma como el profesor negocia su aprendizaje en cada práctica. Por ejemplo, para que el profesor implemente en su enseñanza lo acordado por sus compañeros de profesión durante una junta de academia (práctica de academia), es necesario que él interprete (proceso de participación) y ejecute (proceso de cosificación) esos acuerdos. Dicho de otra manera, para cumplir con los acuerdos escritos en las minutas (objetos limitáneos) generadas durante la junta de academia, se requiere que el profesor asista a su clase (correduría) y tome decisiones pertinentes encaminadas al cumplimiento de esos acuerdos. De igual manera, para que el profesor aborde en su clase los contenidos del programa de estudio que él recibió de las autoridades de su institución al inicio del ciclo escolar (práctica de gestión), es necesario que, previo a sus clases, él los organice (proceso de participación) y los explique a sus estudiantes con la profundidad que él o la institución requiera (proceso de cosificación); es decir, para cumplir con lo establecido en los documentos oficiales (objetos limitáneos) de las instituciones públicas, es necesario que el profesor los cumpla durante su enseñanza; que actúe como corredor entre la práctica de gestión y la de enseñanza.

Al igual que los conceptos de cosificación y participación, los de correduría y objetos limitáneos se complementan mutuamente. Esta complementariedad la propicia, de hecho, el profesor. En efecto, al ser la enseñanza la práctica donde concurren los objetos limitáneos, cosificados en las otras prácticas, el profesor, justo en el momento de impartir su clase, conecta esos objetos por medio de su participación; por medio de su actuar como un corredor entre prácticas. Con base en este análisis, el profesor puede, entonces, concebirse como un corredor que conecta prácticas que suceden fuera del salón de clase (y en las que él se involucra) con la práctica principal de su quehacer docente: enseñar matemáticas. Es justo en su enseñanza donde los procesos de cosificación y participación de cada una de las prácticas fuera del aula cobran sentido. En la práctica de la enseñanza el profesor no solo enseña, sino también aprende de lo que él mismo hizo en otras prácticas. Las cuatro prácticas juntas de la figura 2 forman una comunidad de práctica de la cual el profesor es un integrante. 


\section{CONSIDERACIONES FINALES}

En este ensayo sugerimos analizar la enseñanza de las matemáticas como una práctica que sucede dentro de una comunidad. Hemos propuesto, en concreto, que esta comunidad de práctica se determina por la relación entre una práctica que sucede en el salón de clase (la práctica de enseñanza) y otras prácticas que ocurren fuera de ella, pero que tienen un estrecho vínculo con las intenciones de educar del profesor (estas otras prácticas fueron identificadas como prácticas de academia, de gestión y de capacitación). Esta relación fue caracterizada por el hecho de plantear que al profesor puede considerársele como un corredor. Con esta consideración es posible proponer que el profesor se encarga no solo de llevar a su práctica de enseñanza la información por él conocida en las otras prácticas, sino además de darle sentido a esa información. Más aún, con este análisis insinuamos que es en la práctica de enseñanza donde el profesor da sentido a las participaciones y las cosificaciones realizadas por él en las prácticas de academia, de gestión y de capacitación.

Consideramos que analizar la enseñanza en relación con otras prácticas nos permite considerar la participación del aula como uno de los eslabones de una cadena de participaciones establecidas por el profesor no con sus estudiantes, sino también con sus colegas. De esta forma, un profesor que en una práctica de academia ha expuesto a sus colegas las dificultades de aprendizaje que experimentan sus estudiantes sobre un concepto matemático específico, y ha escuchado de sus colegas sugerencias encaminadas a resolverlos, está en la posibilidad de cambiar sus estrategias didácticas y, también, su discurso explicativo. Así analizado, este discurso explicativo será, en parte, el resultado de una participación ocurrida fuera del aula.

Análisis futuros son necesarios para detallar, en particular, cómo el discurso del profesor es influenciado por las formas distintas en que él se comunica con sus compañeros de profesión en las prácticas de academia, de gestión y de capacitación y, en general, cómo las participaciones y las cosificaciones del profesor en estas prácticas influyen en el acto de enseñar. Las decisiones tomadas por el profesor en su aula pueden ser estudiadas a partir de asuntos como: los acuerdos a los que llegue con sus colegas durante su participación en sus juntas de academia, la manera como logra interpretar la enseñanza de un contenido matemático en sus cursos de capacitación, la directriz que sigue a raíz de su participación en las reuniones con las autoridades de su institución educativa, etcétera. Ahora bien, dado que el profesor es un corredor, podrían 
identificarse prácticas que no sean propias de su quehacer docente pero en las que él está involucrado (por ejemplo, prácticas de índole religiosa, deportiva, comercial, etcétera) y estudiar cómo utiliza el discurso de estas prácticas en el momento de enseñar matemáticas. Con base en la recomendación de Lave (1996) -quien asegura que para conocer si la enseñanza realmente influye en el aprendizaje de los estudiantes es necesario indagar cómo ellos cambian sus formas de participar en distintas comunidades de práctica-, se requiere conocer todas las prácticas en las que esté involucrado el profesor de matemáticas para explorar a detalle la influencia de ellas en su didáctica; es decir, en sus formas de interactuar con sus estudiantes en el momento de abordar los contenidos temáticos de su curso.

\section{Agradecimientos:}

A la Secretaría de Investigación y Posgrado del IPN que con el número de proyecto SIP-20161221 apoyó esta investigación.

\section{REFERENCIAS}

Adler, J., Ball, D., Krainer, K., Lin, F., \& Novotna, J. (2005). Reflections on an emerging field: researching mathematics teacher education. Educational Studies in Mathematics, 60(3), 359-381.

Brousseau, G. (1997). Theory of Didactical Situations in Mathematics. (N. Balacheff, M. Cooper, R. Sutherland, \& S. Warfield, Trads.). London, Great Britain: Kluwer.

Buysse, V., Sparkman, K. L., \& Wesley, P. W. (2003). Communities of practice: Connecting what we know with what we do. Council for Exceptional Children, 69(3), 263-277.

Castorina, J. A. (1996). El debate Piaget-Vigotsky: la búsqueda de un criterio para su evaluación. En J. A. Castorina, E. Ferreiro, M. Kohl de Oliveira, \& D. Lerner, Piaget-Vigotsky: contribuciones para replantear el debate (pp. 9-44). México D.F., México: Paidós.

Chevallard, Y. (1999). L'analyse des pratiques enseignantes en théorie anthropologique du didactique. Recherches en Didactique des Mathématiques, 19(2), 221-266.

Clarà, M. (2017). How instruction influences conceptual development: Vygotsky's theory revisited. Educational Psychologist, 52(1), 50-62. 
Cobb, P., McClain, K., Lamberg, T. D., \& Dean, C. (2003). Situating teachers' instructional practices in the institutional setting of the school and district. Educational Researcher, 32(6), 13-24.

Cole, M., \& Wertsch, J. (2000). Beyond the individual-social antinomy in discussions of Piaget and Vygotsky. Human Development, 39(5), 250-256.

DeVries, R. (2000). Vygotsky, Piaget, and education: A reciprocal assimilation of theories and educational practices. New Ideas in Psychology, 18(1-2), 187-213.

Farnsworth, V., Kleanthous, I., \& Wenger-Trayner, E. (2016). Communities of practice as a social theory of learning: A conversation with Etienne Wenger. British Journal of Educational Studies, 64(2), 139-160.

Gage, N. (2009). A conception of teaching. New York: Springer.

Groth, R. (2015). Working at the boundaries of Mathematics Education and Statistics Education communities of practice. Journal for Research in Mathematics Education, 46(1), 4-16.

Hallinger, P. \& Heck, R. H. (1998). Exploring the principal's contribution to school effectiveness: 1980-1995. School effectiveness and school improvement, 9(2), 157-191.

Hammer, D. (1995). Epistemological considerations in teaching introductory physics. Science Education, 79(4), 393-413.

Hedegaard, M. (1990). The zone of proximal development as basis for instruction. En L. C. Moll (Ed.), Vygotsky and education: Instructional implications and applications of sociohistorical psychology (pp. 349-371). USA: Cambridge University Press.

Hernández-Rojas, G. (1998). Paradigmas en psicología de la educación. México, D.F.: Paidós. Karpov, Y., \& Haywood, H. C. (1998). Two ways to elaborate Vygotsky's concept of mediation: Implications for instruction. American Psychology, 53(1), 27-36.

Kozulin, A. (2000). Instrumentos psicológicos. (G. Sánchez-Barberán, Trad.). Barcelona, España: Paidós.

Kuhn, T. (1971). La estructura de las revoluciones cientificas. (A. Contin, Trad.). México, D.F., México: FCE.

Lave, J. (1996). Teaching, as learning, in practice. Mind, Culture, and Activity, 3(3), 149-164.

Leinhardt, G., \& Greeno, J. G. (1986). The cognitive skill of teaching. Journal of Educational Psychology, 78(2), 75-95.

Lerner, D. (1996). La enseñanza y el aprendizaje escolar. Alegato contra una falsa oposición. En J. A. Castorina, E. Ferreiro, M. Kohl de Oliveira, \& D. Lerner (Eds.), Piaget-Vigotsky: contribuciones para replantear el debate (pp. 69-118). México D.F., México: Paidós.

Manacorda, M. A. (1987). Historia de la educación II. Del 1500 a nuestros días. (Miguel Martí, Trad.). México D.F., México: Siglo XXI. (Obra original publicada en 1983). 
Morgan, C. (2014). Understanding practices in mathematics education: Structure and text. Educational Studies in Mathematics, 87(2), 129-143.

Norton, A., \& D'Ambrosio, B. (2008). ZPC and ZPD: Zones of teaching and learning. Journal for Research in Mathematics Education, 39(3), 220-246.

Peressini, D., Borko, H., Romagnano, L., Knuth, E. \& Willis, C. (2004). A conceptual framework for learning to teach secondary mathematics: A situative perspective. Educational Studies in Mathematics, 56(1), 67-96.

Piaget, J. (1948). To understand is to invent: The future of education. New York, USA: Grossman Publishers.

Pozo, J. I. (1989). Teorías cognitivas del aprendizaje (Sexta ed.). Madrid, España: Morata. Rogalski, J. (2003). Y-a-t-il un pilote dans la classe? Une analyse de l'activité de l'enseignant comme gestion d'un environnement dynamique ouvert. Recherches en Didactique des Mathématiques, 23(3), 343-388.

Roth, W.-M., \& Radford, L. (2010). Re/thinking the zone of proximal development (symmetrically). Mind, Culture, and Activity, 17(4), 299-307.

Schoenfeld, A. (1998). Towards a theory of teaching-in-context. Issues in Education, 4(1), 1-94.

Sensevy, G., Schubauer-Leoni, M.-L., Mercier, A., Ligozat, F., \& Perrot, G. (2005). An attempt to model the teacher's action in the mathematics class. Educational Studies in Mathematics, 59(1-3), 153-181.

Shayer, M. (2003). Not just Piaget; not just Vygotsky, and certainly not Vygotsky as alternative to Piaget. Learning and Instruction, 13(5), 464-485.

Skott, J. (2013). Understanding the role of the teacher in emerging classroom practices: searching for patterns of participation. ZDM, Mathematics Education, 45(4), 547-559.

Skott, J., Moeskaer Larsen, D., \& Hellsten Østergaard, C. (2011). From beliefs to patterns of participation-shifting the research perspective on teachers. Nordic Studies in Mathematics Education, 16(1-2), 29-55.

Vygotsky, L. S. (1978). Mind in Society: The development of higher psychological processes. Massachussets, USA: Harvard University Press.

Walshaw, M. (2008). Developing theory to explain learning to teach. En T. Brown, \& T. Brown (Ed.), The Psychology of Mathematics: A psychoanalytic displacement (pp. 119-137). Rotterdam, The Netherlands: Sense Publishers.

Webb, P. (2001). Piaget: Implications for teaching. Theory into Practice, 19(2), 93-97.

Wenger, E. (2001). Comunidades de práctica: aprendizaje, significado e identidad. (G. Sánchez-Barberán, Trad.). Madrid, España: Paidós. (Obra original publicada en 1998). 
Isaias Miranda y Ana Luisa Gómez-Blancarte

Wenger, E. (2010). Communities of practice and social learning systems: The Career of a Concept. En C. Blackmore (Ed.), Social learning systems and communities of practice (pp. 179-198). New York, USA: Springer.

ISAIAS MIRANDA

Dirección: Calzada Legaria \#694, colonia Irrigación, alcaldía Miguel Hidalgo, Ciudad de México, C.P. 11500

Teléfono: 57296000 Ext. 67779 